\title{
Toward population health literacy, wellbeing, consumer engagement, and information exchange: Developing Omaha System icons for digital platforms
}

\author{
Karen A. Monsen ${ }^{* 1,2}$, Barbara Martinson ${ }^{3}$, Emily C. Lawrence ${ }^{1}$, Taylor A. Maki ${ }^{4}$, Annika E. Stromme ${ }^{1}$, Elizabeth G. \\ Weirich $^{1}$, Karen S. Martin ${ }^{5}$ \\ ${ }^{1}$ School of Nursing, University of Minnesota, Minneapolis, Minnesota, USA \\ ${ }^{2}$ Institute for Health Informatics, University of Minnesota, Minneapolis, Minnesota, USA \\ ${ }^{3}$ College of Design, University of Minnesota, Minneapolis, Minnesota, USA \\ ${ }^{4}$ Department of Speech-Luanguage-Hearing Sciences, University of Minnesota, Minneapolis, Minnesota, USA \\ ${ }^{5}$ Martin Associates, Omaha, Nebraska, USA
}

Received: August 6, 2015

DOI: $10.5430 /$ ijh.v2n1p71
Accepted: November 16, $2015 \quad$ Online Published: November 30, 2015

URL: http://dx.doi.org/10.5430/ijh.v2n1p71

\begin{abstract}
In the digital age, it is critical for both healthcare professionals and consumers to electronically express, receive, and process important health information. This is especially true for populations with language barriers and low literacy. Visual literacy and health literacy enable optimal health communication and wellbeing. A standardized interface terminology is a linguistic tool that can be used to convey health information at the human-computer interface. The Omaha System has been evaluated and found to be a promising standard for use as a simple, standardized terminology that may promote health literacy and communication between healthcare professionals and consumers. However, a method for visually representing health concepts described by the Omaha System has not been developed. The purpose of this study was to develop a complete set of 42 icons that convey the meaning of each of the 42 Omaha System problem concepts as defined by Martin (2005). Design thinking, universal design methods, and informal survey evaluations were used to determine appropriate imagery for the icons. Data-based revisions were incorporated after each of three informal survey evaluations. The resulting set of 42 icons for Omaha System problem concepts is available in the public domain. Future plans are to conduct extensive global evaluation of concept validity and usefulness of the icons across literacy levels.
\end{abstract}

Key Words: Visual literacy, Icons, Omaha System, Health disparities, Wellbeing

\section{INTRODUCTION}

Literacy, once conceptualized as the ability to read and write, is now seen as a more holistic concept that is central to all types of communication. Literacy helps people achieve social wellbeing and self empowerment, and increases the ability to process diverse and complex information from multiple sources. ${ }^{[1-3]}$ Literacy includes the dimensions of health literacy and visual literacy. ${ }^{[2]}$ Furthermore, in a digital world, literacy becomes a multi-modal experience in which the visual pictorial mode may augment or replace the written,

\footnotetext{
*Correspondence: Karen A. Monsen; Email: mons0122@umn.edu; Address: 5-140 Weaver-Densford Hall, 308 Harvard Street SE, Minneapolis, Minnesota, 55455, USA.
} 
linguistic mode. Thus, concepts can be represented as either words or symbols such that linguistic and symbolic modes can become synergistic in support of literacy. ${ }^{[4,5]}$

\subsection{Health literacy}

Health literacy, defined as "the degree to which individuals have the capacity to obtain, process, and understand basic health information and services needed to make appropriate health decisions" $[3,6]$ is a health determinant that is actionable. Health disparities result from low health literacy including barriers to communication that affect health care access, delivery, and outcomes; especially for populations with language barriers and/or low literacy. ${ }^{[3,6-8]}$ The World Health Organization reported that $12 \%$ of Europeans surveyed have inadequate general health literacy and $35 \%$ have problematic health literacy. ${ }^{[7]}$ Technology resources including the internet have been shown to reinforce health disparities. ${ }^{[9]}$ Methods are needed to improve health literacy in the digital age. Visual literacy solutions may be useful to aid in improving health literacy by overcoming barriers to use of technology for consumers with low literacy.

\subsection{Visual literacy}

The critical importance of visual literacy in the digital age is recognized and supported by clinicians and scholars. ${ }^{[2,10]}$ Visual literacy refers to competencies a human being can develop by seeing and simultaneously integrating other sensory experiences that add a new dimension to meaning. The development of visual literacy competencies is part of the normal human learning process. Visual literacy competencies enable persons to discriminate and interpret visible actions, objects, and symbols; and thus comprehend and communicate with others. ${ }^{[11]}$

\subsubsection{Symbols as visual literacy aids}

The use of symbols as visual literacy aids is a growing trend, especially in digital platforms such as computers and hand-held digital devices, where images that symbolize complex concepts can be incorporated as interactive representations, replacing text and providing ease in navigation to resources. ${ }^{[4]}$ In computing, an icon is a quickly comprehensible visual symbol of a concept representing a function or resource that can be accessed digitally. ${ }^{[12-14]}$ Thus symbols have become a visual literacy tool of the digital age when operationalized as icons in digital platforms.

\subsubsection{Socially responsive design to improve visual literacy}

The First Thinks First Manifesto published in 1964 demands that designers must move from commercial aims to projects that improve the human environment. Thus, designers must be involved in interdisciplinary collaborations that will improve wellbeing for people and enhance the places they live, especially for consumers with low literacy who are more vulnerable to health disparities. ${ }^{[7,15,16]}$

\subsection{Use of symbols and icons to aid in visual literacy}

Symbols that aid in visual literacy, especially in wayfinding, are common in public environments. The familiar symbols found in airports, shopping centers, and medical facilities were developed through collaborative work with design professionals and various governmental and commercial agencies such as the American Institute of Graphic Arts (AIGA), and the Society of Experimental Graphic Design (SEGD). ${ }^{[17,18]}$ The AIGA worked with the U.S. Department of Transportation in the development of travel symbols. Additional sets of symbols were developed for recreation, accessibility, and healthcare. While symbols and icons are used widely in wayfinding and digital communication, health researchers and designers are just beginning to develop and test how icons can be used in health care. ${ }^{[19-23]}$

\subsubsection{History of icons in health}

There are well known symbols that have become iconic in health care. Examples include the Caduceus, a red cross, and the characters Rx. ${ }^{[24-26]}$ The Caduceus (snakes entwined around a cross) is a symbol of medicine with origins in Greek mythology. Over the centuries it was loosely tied to healing. It began to be used as a US military symbol for medicine in the late 19 th century. ${ }^{[24]}$ A red cross has become a worldwide icon of medical help. It used by the Red Cross organizations internationally. During wartimes it became a symbol of health and safety. It has been adapted and used for a variety of events and commercial medically-related goods. ${ }^{[25]}$ The characters Rx were used starting in medieval times as a symbol of taking a medicine, and have evolved into an icon for medical prescriptions. ${ }^{[26]}$ While Rx may be understood, the addition of images including a mortar and pestle - tools for preparing drugs - are frequently used with the letters.

\subsubsection{Modern applications of symbols and icons in health} A set of 50 universal health care symbols for wayfinding in medical facilities have been developed and are publicly available. ${ }^{[17]}$ These 50 icons were modeled after the Universal icons developed by members of AIGA, and produced by a collaboration of Hablamos Juntos, SEGD, and the Robert Wood Johnson Foundation. ${ }^{[27]}$ In additiona, a set of human health icons called the Visualization of Concepts in Medicine (VCM), was developed in France. ${ }^{[28]}$ It is intended for use by clinicians to aid in representing and interpretation of complex multidimensional information in medical records. The VCM icons represent all aspects of human health including body systems and diseases, treatments, and responses to treatment. ${ }^{[28]}$ The VCM as applied within electronic health 
records (EHRs) is a form of structured data communication for clinicians such as is needed to improve health care effectiveness and efficiency. In the digital age, methods are needed for both healthcare professionals and consumers to communicate and process important health information using digital platforms. Universal icons could improve such communication, and should be evaluated for their potential to reduce health disparities. To date, no universal icons have been developed for use by consumers in digital platforms such as mobile applications (apps), EHRs, and personal health records (PHRs).

\subsection{Standardized terminologies}

Standardized terminologies are linguistic tools that encode and communicate comparable health care data. ${ }^{[29]}$ Such standardized terminologies enable information retrieval and interoperability across settings and systems, and are increasingly required and embedded within EHRs. ${ }^{[29]}$ The Omaha System is a standardized terminology that may promote health literacy and communication between healthcare professionals and consumers. ${ }^{[30,31]}$ To achieve the goal of evaluating the use of visual representation to support consumer health literacy and simultaneously enable consumers to communicate and document meaningful information in a standardized way, it was necessary to develop universal icons depicting standardized health care concepts.

\subsection{The Omaha System}

The Omaha System is a simple standardized terminology (data capture tool) that is used internationally to communicate health care assessments and services. ${ }^{[30,32]}$ The Omaha System in its linguistic mode has been shown to be useful for consumer communication about needs and strengths. ${ }^{[30,33,34]}$ Preliminary evaluation showed that Omaha System concepts were amenable to visual representation. ${ }^{[35]}$ For purposes of describing wellbeing and a whole-person perspective, it is especially critical to note that both strengths and problems have been shown in relationship to the 42 Omaha System problem concepts, and that the model of Wellbeing as described by Kreitzer $^{[36]}$ has been mapped to the Omaha System. ${ }^{[30,34]}$

Because its terms are defined and arranged taxonomically in a robust information model, the Omaha System is amenable to adoption as a knowledge representation tool within digital platorms. ${ }^{[30,32]}$ Data generated by use of the Omaha System has been used extensively in healthcare quality research. ${ }^{[32,37]}$ The Omaha System has three components that are psychometrically sound intruments, the Problem Classification Scheme, The Intervention Scheme, and the Problem Rating Scale for Outcomes. ${ }^{[30]}$ These three components interrelate because each is structured around the common core set of 42 problems concepts.

Published by Sciedu Press

\subsubsection{Problem Classification Scheme}

The Problem Classification Scheme classifies health and healthcare terms in a taxonomic structure within 42 structured problem concepts that are further classified within four Domains: Environmental, Psychosocial, Physiological, and Health-related Behaviors (see Table 1). Each problem concept is defined and has a set of unique signs/symptoms. Definitions are intended to be neutral (e.g. Skin is defined as the natural covering of the body). In its linguistic mode, the terms of the Problem Classification Scheme enable healthcare communication regarding problems, signs/symptoms, and strengths. The data from the Problem Classification Scheme can be used for aggregate reporting of population health concerns, and statistical modeling of health characteristics. ${ }^{[30]}$ Consumers may be able to describe strengths and needs relative to any of the 42 problem concepts. ${ }^{[30,33,34]}$

\subsubsection{Intervention Scheme}

The Intervention Scheme describes healthcare activities in a three-level hierarchy relative to Omaha System problem concepts. The three levels consist of the action (Category), the defined detail (Target), and the additional detail that may be customized (Care description). There are 4 Categories: Teaching, guidance, and counseling, Treatments and procedures, Case management, and Surveillance (see Table 2); and 75 Target terms arranged alphabetically (e.g. feeding procedures, medication administration, and skin care). Linguisitically, the terms of the Intervention Scheme enable description of consumer needs, multi-dsciplinary care planning, intervention documentation, and clinical decision support, including evidence-based standardized care plans. ${ }^{[38]}$ The data generated using the Intervention Scheme can be employed in intervention description and effectiveness studies. ${ }^{[30]}$ Consumers may be able to engage in collaborative care planning using terms from the Intervention Scheme. ${ }^{[30,31]}$

\subsubsection{Problem Rating Scale for Outcomes}

The Problem Rating Scale for Outcomes consists of three valid, reliable measures of problem concept-specific Knolwedge, Behavior, and Status. These Likert-type ordinal scales serve to normalize and level healthcare assessments relative to any Omaha System problem concept on a scale of 1-5 ( $1=$ lowest to $5=$ highest $)$. Linguistically, the scales describe degrees of problem-specific Knowledge, Behavior, and Status (see Table 3) that can describe clinical and/or self-assessments and goals. Data generated from use of the Problem Rating Scale for Outcomes can be employed in benchmarking studies and outcomes measurement analyses including predictive modeling and population health outcome research. ${ }^{[30]}$ Consumers may be able to rate the severity of their sign/sypmtoms using the Problem Rating Scale for Outcomes. ${ }^{[30]}$ 


\subsection{Importance of the problem}

Consumers with language barriers and low literacy may be disproportionately challenged to access health information and communicate their strengths and needs in the digital age. ${ }^{[2,10]}$ Technology offers new opportunities for visual literacy support including data collection by increasing use of tools that promote visual literacy and support communication between clinicians and consumers with low literacy and/or language barriers. ${ }^{[4,5]}$ The Omaha System has been shown to be a powerful information model both linguistically and as structured data; however its value in symbolic visual mode is unexplored. There is potential to visualize Omaha System concepts symbolically in icons for digital platforms; and thus to support health literacy through visual literacy.

Table 1. Omaha System Problem Classification Scheme Domain, Problem, and Signs/Symptom terms ${ }^{[30]}$

\begin{tabular}{|c|c|c|}
\hline Omaha System Domain & Domain Definition & $\begin{array}{l}\text { Problem concepts (number of signs/symptoms; example of a } \\
\text { sign/symptom) }\end{array}$ \\
\hline Environmental Domain & $\begin{array}{l}\text { Material resources and physical surroundings both } \\
\text { inside and outside the living area, neighborhood, and } \\
\text { broader community. }\end{array}$ & $\begin{array}{l}\text { Income (5; difficulty buying necessities) } \\
\text { Sanitation (11; presence of mold) } \\
\text { Residence (14; exposed wiring) } \\
\text { Neighborhood/workplace safety (10; high pollution level) }\end{array}$ \\
\hline Psychosocial Domain & $\begin{array}{l}\text { Patterns of behavior, emotion, communication, } \\
\text { relationships, and development. }\end{array}$ & $\begin{array}{l}\text { Communication with community resources (11; language barrier) } \\
\text { Social contact (3; uses health care provider for social contact) } \\
\text { Role change (3; involuntary role reversal) } \\
\text { Interpersonal relationship (8; prolonged, unrelieved tension) } \\
\text { Spirituality (4; disrupted spiritual rituals) } \\
\text { Grief (4; difficulty expressing grief responses) } \\
\text { Mental health (17; difficulty managing stress) } \\
\text { Sexuality (8; difficulty expressing intimacy) } \\
\text { Caretaking/parenting (9; abusive) } \\
\text { Neglect (6; lacks emotional nurturance, support) } \\
\text { Abuse (8; harsh, excessive discipline) } \\
\text { Growth and development (4; age-inappropriate behavior) }\end{array}$ \\
\hline Physiological Domain & Functions and processes that maintain life. & $\begin{array}{l}\text { Hearing (5; difficulty hearing high frequency sounds) } \\
\text { Vision (8; difficulty seeing close objects) } \\
\text { Speech and language (6; limited enunciation/clarity) } \\
\text { Oral health (7; caries) } \\
\text { Cognition (10; limited recall of recent events) } \\
\text { Pain (6; expresses discomfort/pain) } \\
\text { Consciousness (4; unresponsive) } \\
\text { Skin (10; rash) } \\
\text { Neuro-musculo-skeletal function (13; limited range of motion) } \\
\text { Respiration (10; abnormal breath sounds) } \\
\text { Circulation (16; abnormal blood pressure reading) } \\
\text { Digestion-hydration (10; nausea/vomiting) } \\
\text { Bowel function (7; abnormal bowel sounds) } \\
\text { Urinary function (8; difficulty initiating urination) } \\
\text { Reproductive function (7; infertility) } \\
\text { Pregnancy (6; difficulty coping with body changes) } \\
\text { Postpartum (6; postpartum complications) } \\
\text { Communicable/infectious condition (8; infection) }\end{array}$ \\
\hline $\begin{array}{l}\text { Health-related Behaviors } \\
\text { Domain }\end{array}$ & $\begin{array}{l}\text { Patterns of activity that maintain or promote } \\
\text { wellness, promote recovery, and decrease the risk of } \\
\text { disease }\end{array}$ & $\begin{array}{l}\text { Nutrition (11; improper feeding schedule for age) } \\
\text { Sleep and rest patterns (8; insomnia) } \\
\text { Physical activity (3; sedentary lifestyle) } \\
\text { Personal care (9; foul body odor) } \\
\text { Substance use (9; abuses alcohol) } \\
\text { Family planning (6; difficulty obtaining family planning methods) } \\
\text { Health care supervision (7; inconsistent source of health care) } \\
\text { Medication regimen (8; fails to obtain refills appropriately) }\end{array}$ \\
\hline
\end{tabular}

\subsection{Purpose}

The long term goal of our research is to evaluate the effectiveness of icons in reducing health disparities. Our immediate goal is to support visual literacy as a way of enhancing health communication for all users in digital platforms such as PHRs and EHRs based on the three components of the
Omaha System. In order to achieve robust representation of Omaha System concepts for further evaluation among consumers with language barriers and low literacy, it was necessary to develop icons depicting Omaha System concepts. The purpose of this study was to develop a complete set of 42 icons that convey the meaning of each of the 42 Omaha System problem concepts as defined by Martin. ${ }^{[30]}$ 
Table 2. Omaha System Intervention Scheme Category terms with definitions ${ }^{[30]}$

\begin{tabular}{ll}
\hline Omaha System Category & Category Definition \\
\hline $\begin{array}{l}\text { Teaching, guidance, and } \\
\text { counseling }\end{array}$ & $\begin{array}{l}\text { Activities designed to provide information and materials, encourage action and responsibility for self-care and } \\
\text { coping, and assist the individual/family/community to make decisions and solve problems. } \\
\text { Treatments and procedures } \\
\text { Technical activities such as wound care, specimen collection, resistive exercises, and medication prescriptions that } \\
\text { are designed to prevent, decrease, or alleviate signs and symptoms of the individual/family/community. }\end{array}$ \\
Activities such as coordination, advocacy, and referral that facilitate service delivery, improve communication among \\
health and human service providers, promote assertiveness, and guide the individual/family/community toward use of \\
appropriate resources. \\
Activities such as detection, measurement, critical analysis, and monitoring intended to identify the \\
individual/family/community's status in relation to a given condition or phenomenon.
\end{tabular}

Table 3. Omaha System Problem Rating Scale for Outcomes with definitions ${ }^{[30]}$

\begin{tabular}{|c|c|c|c|c|c|}
\hline Scale (definition) & 1 & 2 & 3 & 4 & 5 \\
\hline $\begin{array}{l}\text { Knowledge: Ability of the client to remember and } \\
\text { interpret information }\end{array}$ & No knowledge & Minimal knowledge & Basic knowledge & Adequate knowledge & Superior knowledge \\
\hline $\begin{array}{l}\text { Behavior: Observable responses, actions, or } \\
\text { activities of the client fitting the occasion or purpose }\end{array}$ & $\begin{array}{l}\text { Not appropriate } \\
\text { behavior }\end{array}$ & $\begin{array}{l}\text { Rarely appropriate } \\
\text { behavior }\end{array}$ & $\begin{array}{l}\text { Inconsistently } \\
\text { appropriate behavior }\end{array}$ & $\begin{array}{l}\text { Usually appropriate } \\
\text { behavior }\end{array}$ & $\begin{array}{l}\text { Consistently } \\
\text { appropriate behavior }\end{array}$ \\
\hline $\begin{array}{l}\text { Status: Condition of the client in relation to objective } \\
\text { and subjective defining characteristics }\end{array}$ & $\begin{array}{l}\text { Extreme signs/ } \\
\text { symptoms }\end{array}$ & $\begin{array}{l}\text { Severe signs/ } \\
\text { symptoms }\end{array}$ & $\begin{array}{l}\text { Moderate signs/ } \\
\text { symptoms }\end{array}$ & $\begin{array}{l}\text { Minimal signs/ } \\
\text { symptoms }\end{array}$ & No signs/ symptoms \\
\hline
\end{tabular}

\section{METHOD}

The icon development study employed standard participatory design methods in a multi-stage design process, incluing 1) design thinking, 2) universal design methods, and 3) informal survey evaluations, in order to determine appropriate imagery and develop the icons. ${ }^{[39-43]}$ Each of the methods was applied in iterative steps as needed to achieve consensus among the designers on the final icon designs. These methods were chosen because each is sensitive to the needs of consumers in communicating ideas through design. Design thinking is an inclusive, inter-disciplinarly human intelligence process that draws out novel insights to solve problems. ${ }^{[39]}$ Universal and inclusive design is aimed at ensuring that all people have access to essential communications. ${ }^{[40,41]}$ Finally, informal survey research was imployed to engage the general public in the design. Informal survey research taps into the collective intelligence of the public at large to complete a task; taking advantage of valid opportunities to collect information. This cost-effective approach does not claim statistical or sampling precision. It can be used to identify issues and impressions as a starting point for more comprehensive study. ${ }^{[42,43]}$

\subsection{Participant characteristics}

Throughout the study the designers engaged the public in evaluating prototype images. Participants in the prototype testing phase were adult conference attendees at the 2014 International Visual Literacy Association Conference, the 142nd Annual Meeting of the American Public Health Association, and the 2015 Omaha System International Confer- ence. They self-selected into the study by participating in an interactive session in which the revised icon prototypes were presented during poster and oral presentations, and by providing feedback in structured responses and discussion of issues in visual concept representation.

\subsection{Sampling procedures}

The sampling procedure was purposive due to the intentional selection of national and international conferences. It was a convenience sample because participants self-selected into the study by attending conference sessions and completing evaluations.

\subsubsection{Sample size}

Sample size was based on previous literature related to icon development, which ranged from 20-242 participants per test. ${ }^{[19-23]}$

\subsubsection{Measures}

Worksheets were developed to assess the ability of participants to discriminate between visual representations of selected Omaha System concepts and associate the correct Omaha System terms with visual representations. Correctly naming an icon or matching the icon to the intended Omaha System concept term constituted a correct answer. The percentages of correct responses were calculated for each test. Additionally, one test evaluated the use of color for improving interpretation of selected icons, with yes indicating that color within the icon improved interpretation, vs. no indicating that color within the icon did not improve interpretation. 


\subsection{Icon development process}

The design thinking process, incorporating elements of universal design, was used to develop ideas and images for the 42 Omaha System concepts. The designers consisted of design expert (BM), Omaha System expert (KAM), and four research assistants (EL, TM, AS, and EW). The designers worked closely over a period of 18 months. Four research assistants drafted prototype images over a period of three months, based on concept definitions, literature reviews, and internet searches. They networked with persons in the real world, both with and without a health care background, to problem-solve issues and refine the prototypes. The initial prototype icons consisted of original sketches, stick figures, photographs, and electronic drawings. The prototypes were compiled into a document for presentation purposes. Then the research assistants and an Omaha System expert met weekly for three months, discussed the meanings of each concept, and reviewed prototype images drafted by the research assistants. The design expert then reviewed the 42 prototype images and the entire team discussed strategies for formalizing the images.

Next, the design concept formalized the images, incorporating elements of universal design and maximizing use of existing symbols. Throughout the study, the designer and team informally sought opinions of the general public in the development of visual representations of the 42 Omaha System concepts. The 42 prototypes were revised and evaluated sequentially at three national/international conferences by audiences with interest and expertise in visual literacy, public health, and the Omaha System. The informal survey evaluations were conducted using paper worksheets that elicited participant insights and feedback about validity of the icons by matching icons and concept names. Thirty-seven problem concepts were evaluated in one or more of the tests (exceptions were Caretaking/parenting, Communicable/infectious condition, Medication regimen, Physical activity, and Sexuality). Written and verbal comments were documented by the designers. Materials developed for tests included grouping icons to evaluate discernment between similar symbols (e.g. all "heads").

Preliminary work included novel designs for all icons, incorporating four colors (green, pink, blue, and orange) corresponding to the Domains of the Omaha System. Prior to the first conference, the designer and team members reached agreement on standard format and style in accordance with the SEGD ${ }^{[18]}$ design for health wayfinding. This decision enabled reuse of original or slightly modified SEGD symbols for 23 Omaha System problem concepts, and used black and whte for all design elements. Between the second and third conference, the designer incorporated color (using black, white, and red) and anatomical design based on $\mathrm{VCM}^{[28]}$ icons. The participants from the first and second conference evaluated 31 icons, of which 13 were finalized. The participants from the third conference evaluated 21 icons as well as the role of color in improving interpretability of the icons after which 38 icons were finalized. After the final test, the designer further developed four icons (Pain, Mental health, Consciousness, and Interpersonal relationship) and reached consensus on the set of 42 Omaha System icons. Many concepts required multiple iterations and simplification of the visual representation relative to the concept definition. Ultimately, the designer and team members selected the best fit between SEGD or VCM prototypes, informal survey evaluation feedback, and design team opinion.

\section{RESULTS}

An iterative process of formally designing, testing, and revising prototype designs was repeated until designer and team members reached consensus for each of the 42 concepts. Percentages of correct responses were used to analyze participant responses at various stages of icon development.

\subsection{Number of participants}

Participants in the testing phase included a total of 219 respondents, with an average of 54 responses from each. Each conference had attendees from numerous countries. Participant characteristics were not collected.

\subsection{Participant responses}

\subsubsection{Matching and color use tests}

Participant evaluations of various iteratons of the icons are presented in (see Tables 4-6). The majority of respondents indicated that use of color was helpful in interpreting selected icons (see Tables 4-6). Tables 4-6 provide all Omaha System Problem concepts and their definitions, the final icon design, percentages of respondents who agreed that color use helped participants understand the icon and percentages of correct matches in informal survey evaluations.

\subsubsection{Written responses}

Of 70 brief comments abstracted from worksheets, $3(4.3 \%)$ affirmed the icon(s), 20 (28.6\%) made suggestions for changes or imprvements (e.g. "add a drop of water"), and $47(67.1 \%)$ indicated confusion about the intended meaning of the icon. Of the latter, 23 (48.9\%) indicated inability to distinguish between 2 or more similar icons (e.g. Consciousness vs. Cognition), 18 (38.3\%) offered alternative interpretations of the icon (e.g. "is that a lightening bolt?"), and $6(12.8 \%)$ indicated that the respondent was unablee to interpret the icon (e.g. "makes no sense"). 
Table 4. Summary of Omaha System Problem concepts from the Environmental and Psychosocial Domains, with definitions, the final icon design, and results of informal survey evaluation tests. Definitions from Martin. ${ }^{[30]}$

\section{Environmental Domain}

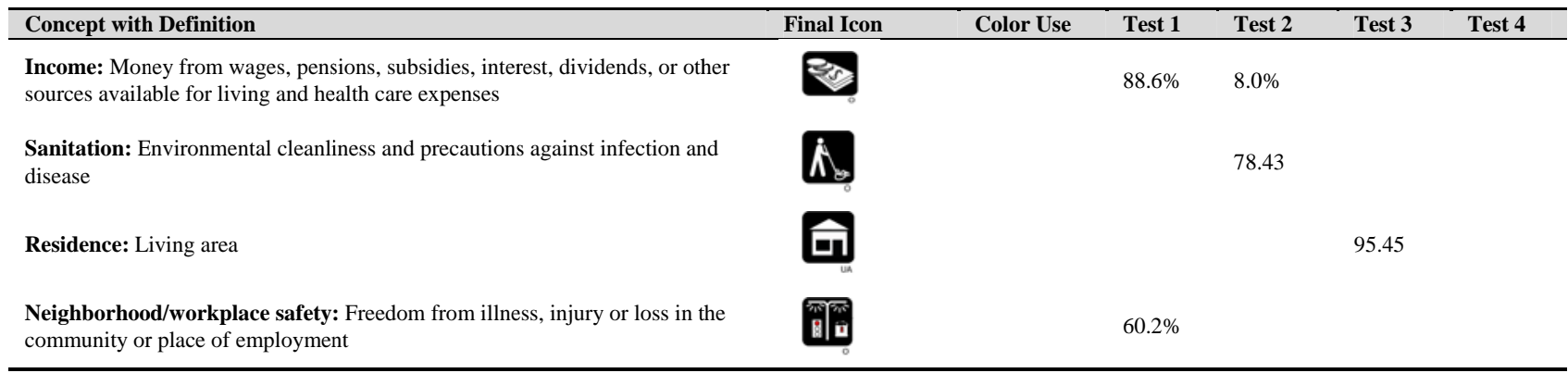

\section{Psychosocial Domain}

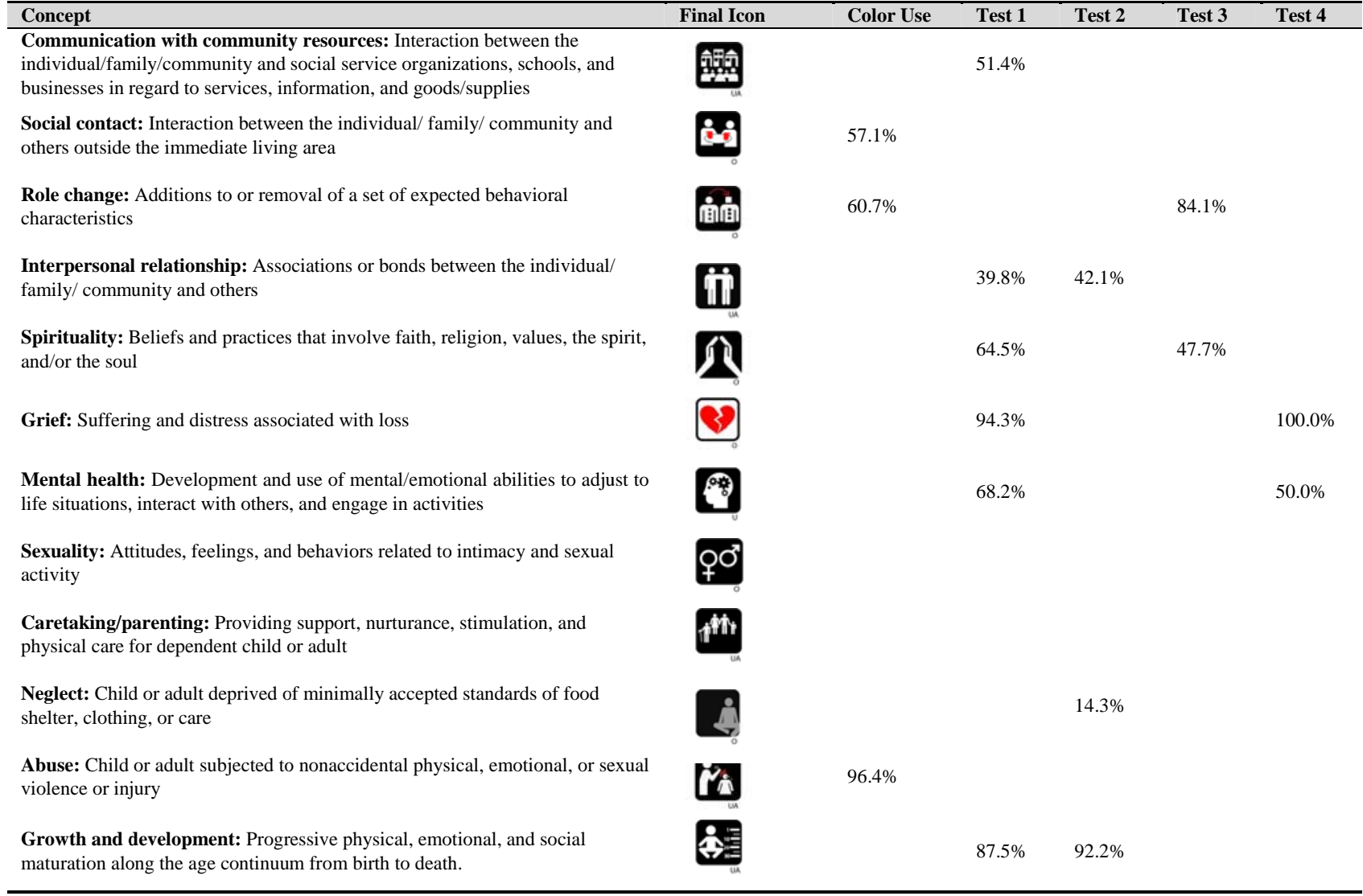

Note. $\mathrm{U}=$ Universal, $\mathrm{UA}=$ Universal Adapted, $\mathrm{O}=$ Original

\subsection{Omaha System icons in four domains}

The Omaha System icons are available on-line. ${ }^{[38]}$ Tables 4-6 summarize study findings and includes the final icon image, the problem concept definition, and results of color use and matching tests. Each icon had multiple iterations and the tests were conducted at various stages of icon design. Due to space limitations, only the final icons are shown. Each icon includes a notation to denote whether it is original (O), universal (U), or an adaptation of a universal icon (UA).

\section{Discussion}

In this study, icons visually representing the 42 concepts of the Omaha System were developed using design thinking, universal design principles, and informal survey evaluations. Data-based revisions were incorporated after each test. Design principles developed during the study informed final revisions to ensure uniform presentation of various aspects of the concepts. The resulting icons are available in the public domain. Further research is needed to refine the icons for use globally and across literacy levels. 
Table 5. Summary of Omaha System Problem concepts from Physiological Domain, with definitions, the final icon design, and results of informal survey evaluation tests. Definitions from Martin. ${ }^{[30]}$

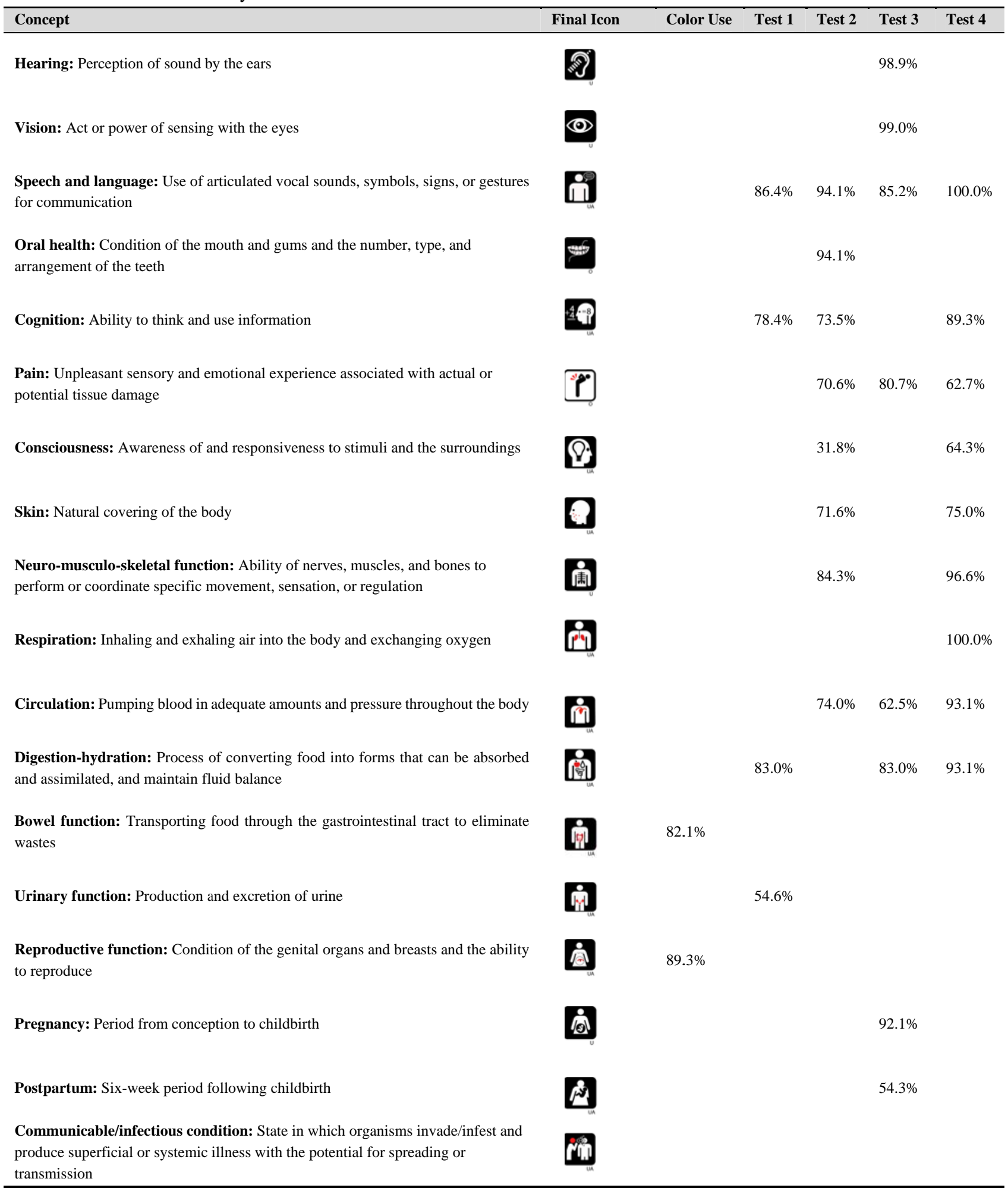

Note. $\mathrm{U}=$ Universal, $\mathrm{UA}=$ Universal Adapted, $\mathrm{O}=$ Original

From the test responses and comments, it is clear that the visual representation of complex healthcare concepts is challenging. While most consumers might readily understand an image of a house as signifying a place to live (the Residence concept), there is a greater challenge in depicting some of the less concrete concepts such as Pain, Mental 
health, Consciousness, and Interpersonal relationship. It was particularly difficult to ensure that two or more icons depicting conceptually or anatomically similar items were visually distinct (e.g. closely related symbolism for Cognition, Consiousness, and Mental health). Other concepts were more difficult because they were complex and multidimensional, such as Neuro-musculo-skeletal function and Neighborhoodworkplace safety. These observations align with literature describing the VCM development process, and reinforce the need for extensive involvement of users including consumers in the icon development process. ${ }^{[18,28]}$

Table 6. Summary of Omaha System Problem concepts from the Health-related Behaviors Domain, with definitions, the final icon design, and results of informal survey evaluation tests. Definitions from Martin. ${ }^{[30]}$

\begin{tabular}{l}
\hline Concept \\
Nutrition: Select, consume, and use food and fluids for energy, maintenance, growth, and \\
health \\
Sleep and rest patterns: Periods of suspended motor and sensory activity and periods of \\
inactivity, repose, or mental calm \\
Physical activity: State or quality of body movements during daily living \\
Personal care: Management of personal cleanliness and dressing \\
$\begin{array}{l}\text { Substance use: Consumption of medicines, recreational drugs, or other materials likely to } \\
\text { cause mood changes and/or psychological/physical dependence, illness, and disease }\end{array}$ \\
$\begin{array}{l}\text { Family planning: Practices designed to plan and space pregnancy within the context of values, } \\
\text { attitudes, and beliefs }\end{array}$ \\
Health care supervision: Management of the health care treatment plan by health care \\
providers \\
Medication regimen: Use or application of over-the-counter and prescribed/recommended \\
medications and infusions to meet guidelines for therapeutic action, safety, and schedule
\end{tabular}

Note. $\mathrm{U}=$ Universal, $\mathrm{UA}=$ Universal Adapted, $\mathrm{O}=$ Original

As described in the literature, we found that design thinking and informal survey methods were keys to incorporating public opinion as an essential aspect of the design process. ${ }^{[39-43]}$ Design team immersion in this project coupled with external feedback from the general public enabled a rich dialogue that was informed by potential future consumers, Omaha System experts, designer, and team members. The iterative evaluation process enabled step by step refinement of both the images themselves and aspects of the design approach, so that design principles for the icons could be refined and applied across the entire set. Interactive presentations as an informal survey evaluations enabled designer and team membes to test the evolving icons over several iterations both with the general public and at national or international conferences. Future research will leverage public and social media to further test the icons globally across populations and languages.

The goal of this study was to develop a set of icons to visually represent Omaha System concepts; and the future goal is that the Omaha System icons could be universally under- stood and used by all consumers. However, because cultural, language, and literacy differences may influence icon interpretation, ${ }^{[4,5]}$ further refinement of the icons is needed to ensure universal knowledge representation across cultures, languages, and literacy levels. Toward that end, surveys evaluating the meaning of these icons are underway in many languages. Additional revisions will be made based on the findings of these studies, with the goal of having a single universal set of final icons that can be used in digital platforms globally in the same way use of the Omaha System enables structured knowledge representation in many languages. ${ }^{[32]}$ A limitation of this study was lack of testing by literacy level. Further research should focus on use of the icons to describe both strengths and needs of low literacy populations.

The implications and research agenda set forth from this research are many. The icons will be available in the public domain for use in digital platforms. Having a complete set of icons representing health and healthcare concepts based on the Omaha System ontology launches a new era in interoperability that does not rely solely on linguistic representation 
for health literacy and consumer engagement. Consumers who previously would be unable to inform caregivers and clinicians of their problems and strengths may be empowered to communicate in a more robust, rigorous way, and thus improve clinical outcomes. Such communication could enable structured data capture that aligns with the data infrastructure of clinical databases, and thus lead to improved consumer-centered outcomes analysis using large datasets of consumer-generated data. Furthermore, given that the Omaha System concepts have been successfully used to classify strengths, consumers with low literacy may be able to use these icons to provide data about their positive attributes and wellbeing as well as health problems, and be enabled to more fully engage and participate in strengths-based assessments and care planning. ${ }^{[32,33]}$
This study used design thinking, universal design principles, and informal survey evaluation to translate clinical health concepts into icons for use in electronic communication. A complete set of icons for 42 Omaha System problem concepts is now available in the public domain. Further research is needed to refine the icons for use globally and across literacy levels. Use of the icons in research will enable evaluation of visual literacy as a component of health literacy; and examine the potential of icon use to reduce health disparities.

\section{ACKNOWLEDGEMENTS}

The University of Minnesota College of Design, School of Nursing Center for Nursing Informatics, and the Omaha System Partnership provided institutional support for this research. Special thanks to Yu Jin Kang and Ryan Schneider.

\section{REFERENCES}

[1] Freire P. Education for Critical Consciousness. New York: Continuum; 1973. $140 \mathrm{p}$.

[2] Glassman P. Accessed from the National Network of Libraries of Medicine [Internet]. Health Literacy. [cited 2015 November 18] Available from: http://nnlm.gov/outreach/consumer/hlthl it.html

[3] Institute of Medicine of the National Academies. Health literacy: A prescription to end confusion. Washington, DC: Institute of Medicine; 2004. $350 \mathrm{p}$.

[4] Daiute C. Educational uses of the digital world for human development. In Butler-Kisber, L. ed. Teaching and learning in a digital world: Possibilities and challenges. LEARNing Landscapes. 2013; 6(2): 63-84.

[5] Hull G, Nelson M. Locating the semiotic power of multimodality. Writ Commun. 2005; 22(2): 224-61. http://dx.doi.org/10.11 $77 / 0741088304274170$

[6] Dewalt DA, Berkman ND, Sheridan S, et al. Literacy and health outcomes: A systematic review of the literature. J Gen Intern Med 2004; 19(12): 1228-39. PMID:15610334. http://dx.doi.org/1 $0.1111 / j .1525-1497.2004 .40153 . x$

[7] Kickbusch I, Pelikan JM, Apfel F, et al. Health literacy: The solid facts. Geneva: World Health Organization; 2013. 86 p.

[8] Berkman ND, Sheridan SL, Donahue KE, et al. Health literacy interventions and outcomes: An updated systematic review [Internet] Rockville(MD): Agency for Healthcare Research and Quality (US); (2011 Evidence Reports/Technology Assessments, No. 199.). [cited 2015 November 18]. Available from: http://www.ncbi.nlm.nih .gov/books/NBK82434/

[9] Neter E, Brainin E. eHealth Literacy: Extending the Digital Divide to the Realm of Health Information. Journal of Medical Internet Resesarch. 2012; 14(1): e19. http://dx.doi.org/10.2196/jmir 1619

[10] Lee TW, Lee SH, Kim HH, et al. Effective intervention strategies to improve health outcomes for cardiovascular disease patients with low health literacy skills: A systematic review. Asian Nursing Research 2013; 6: 128-136. PMID:25031114. http://dx.doi.org/10.10 $16 / j$.anr.2012.09.001
[11] Fransecky RB, Debes JL. Visual literacy: A way to learn - A way to teach. Washington DC: Association for Educational Communications and Technology; 1972.

[12] Tech terms. Tech terms computer dictionary [Internet]. [cited 2015 November 18]. Available from: http://techterms.com/defini tion/icon

[13] Wiley Corporation. Information Technology for Management. 1997. [cited 2015 November 18]. Available from: http://www.wiley . com/college/turban/glossary.html

[14] Wikipedia. Icon (computing) [Internet]. 2015. [cited 2015 November 18]. Available from: https://en.wikipedia.org/wiki/Ic on_(computing)

[15] Clarkson PJ, Langdon P, Robinson P. Designing accessible technology. London, UK: Springer; 2006. 250 p. http://dx. doi .org/1 $0.1007 / 1-84628-365-5$

[16] Margolin V, Margolin S. A "Social Model" of Design: Issues of Practice and Research. Des Issues. 2002; 18(4): 24-30. http: //dx.doi.org/10.1162/074793602320827406

[17] American Institute of Graphic Arts (AIGA). Symbol signs. [cited 2015 November 18]. Available from: http://www .aiga.org/sym bol-signs/

[18] Society for Experimental Graphic Design (SEGD). Healthcare symbols. [cited 2015 November 18]. Available from: https://segd.o $\mathrm{rg} / \mathrm{healthcare-symbols}$

[19] Choi J. Development and pilot test of pictograph-enhanced breast health-care instructions for community-residing immigrant women. Int J Nurs Pract. 2012; 18(4): 373-78. PMID:22845637. http: //dx.doi.org/10.1111/j.1440-172X.2012.02051.x

[20] Griffon N, Kerdelhué G, Hamek S, et al. Design and usability study of an iconic user interface to ease information retrieval of medical guidelines. J Am Med Inform Assoc. 2014 Oct; 21(e2): e270-7. http://dx.doi.org/10.1136/amiajnl-2012-001548

[21] Kim H, Nakamura C, Zeng-Treitler Q. Assessment of Pictographs Developed Through a Participatory Design Process Using an Online Survey Tool. J Med Internet Res. 2009 Jan-Mar; 11(1): e5. PMID:19275981. http://dx.doi.org/10.2196/jmir.1129

[22] Kripalani S, Robertson R, Love-Ghaffari MH, et al. Development of an illustrated medication schedule as a low-literacy patient education 
tool. Patient Educ Couns. 2007 Jun; 66(3): 368-77. PMID:17344015. http://dx.doi.org/10.1016/j.pec.2007.01.020

[23] Lalloo C, Stinson JN, Hochman JR, et al. Adapting the Iconic Pain Assessment Tool Version 2 (IPAT2) for adults and adolescents with arthritis pain through usability testing and refinement of pain quality icons. Clinical J Pain. 2013; 29(3); 253-64. PMID:22936076. http://dx.doi.org/10.1097/AJP.0b013e318250e655

[24] Wilcox R. The symbol of modern medicine: Why one snake is more than two. Ann Intern Med. 2003; 138(7): 673-7. PMID:12693891. http://dx.doi.org/10.7326/0003-4819-1 38-8-200304150-00016

[25] Red Cross [Internet]. [cited 2015 November 18]. Available from: http://www.redcross.org/about-us/history

[26] Dowse R, Ehler M. Pictograms in pharmacy. International Journal of Pharmacy Practice. 1998; 6: 109-18. http://dx.doi.org/10.11 11/j.2042-7174.1998.tb00924.x

[27] Hablamos Juntos [Internet]. [cited 2015 November 18]. Available from: http://www.hablamosjuntos.org/signage/PDF /HJWorkbookFinalwAttachments.pdf

[28] VCM: An Iconic Language for the Visualization Concepts in Medicine [Internet]. [cited 2015 November 18]. Available from: http://vcm.univ-paris13.fr/content/clinical-g uidelines

[29] Chute CG. Clinical Classification and Terminology: Some History and Current Observations. J Am Med Inform Assn. 2000; 7(3): 298303. PMID:10833167. http://dx.doi.org/10.1136/jamia.2 000.0070298

[30] Martin KS. The Omaha System: A key to practice, documentation, and information management (Reprinted 2nd ed.). Omaha, NE: Health Connections Press; 2005. 484 p.

[31] Monsen KA, Westra BL, Paitich N, et al. Developing a shared personal health record for elders and providers: Technology and content. J Gerontol Nurs. 2012; 38(7): 21-5. PMID:22715956. http://dx.doi.org/10.3928/00989134-20120605-03

[32] Omaha System. The Omaha System: Solving the clinical datainformation puzzle [Internet]. [cited 2015 November 18]. Available from: http://omahasystem.org

[33] Monsen KA, Holland DE, Fung-Houger PW, et al. Seeing the whole person: Feasibility of using the Omaha System to describe strengths of older adults with chronic illness. Research and Theory for Nursing Practice. 2014; 28(4): 299-315. PMID:25577860. http://dx.doi.org/10.1891/1541-6577.28.4.299

[34] Monsen KA, Peters J, Schlesner S, et al. The gap in Big Data: Getting to wellbeing, strengths, and a whole person perspective. Global Advances. 2015; 4(3): 31-39. PMID:25984416. http://dx.doi.o $\mathrm{rg} / 10.7453 / \mathrm{gahmj} .2015 .040$

[35] Monsen KA, Martinson B, Stromme A, et al. Development of a visual literacy standard to promote health literacy using the Omaha System. Paper presented at the 140th American Public Health Association Annual Meeting, New Orleans. 2014.

[36] Kreitzer MJ, Koithan M. Integrative nursing. Oxford Press: Cary, NC; 2014. 608 p. http://dx.doi.org/10.1093/med/9780199 860739.001 .0001

[37] Omaha System Partnership. The Omaha System Partnership for Knowledge Discovery and Health Care Quality [Internet]. [cited 2015 November 18]. Available from: http://omahasystempartn ership.org

[38] Omaha System Guidelines. Omaha System Icons Study [Internet] [cited 2015 November 18]. Available from: http://omahasyste mguidelines . org/?p=295

[39] Brown T. Change by design: How design thinking transforms organizations and inspires innovation. New York: HarperCollins; 2009. $264 \mathrm{p}$.

[40] Papanek V. Design for the Real World: Human Ecology and Social Change. (2nd ed.). New York, NY: Van Nostrand Reinhold Company; 1984. 394 p.

[41] Reed DJ, Monk A. Design for inclusion, pp 53-63 in Clarkson J, Langdon P. Designing accessible technology. Springer-Verlag: London; 2006. $251 \mathrm{p}$.

[42] Marsland N. Informal Survey Methods. Natural Resources Institute (NRI) of the University of Greenwich, U.K. 2001. [cited 2015 November 18]. Available from: www.nri.org/projects/publication s/bpg/bpg10.pdf

[43] Franzel S, Crawford EW. Comparing formal and informal survey techniques for farming systems research: A case study from Kenya. Agricultural Administration and Extension. 1987; 27(1): 13-33. http://dx.doi.org/10.1016/0269-7475(87)90007-9 